\title{
Enhance RSS-Based Indoor Localization Accuracy by Leveraging Environmental Physical Features
}

\author{
Peng Xiang, Peng Ji, and Dian Zhang (D) \\ Guangdong Province Key Laboratory of Popular High Performance Computers, Shenzhen University, Shenzhen, China \\ Correspondence should be addressed to Dian Zhang; zhangd@szu.edu.cn
}

Received 25 February 2018; Accepted 4 May 2018; Published 9 July 2018

Academic Editor: Wei Wang

Copyright (C) 2018 Peng Xiang et al. This is an open access article distributed under the Creative Commons Attribution License, which permits unrestricted use, distribution, and reproduction in any medium, provided the original work is properly cited.

\begin{abstract}
Indoor localization technologies based on Radio Signal Strength (RSS) attract many researchers' attentions, since RSS can be easily obtained by wireless devices without additional hardware. However, such technologies are apt to be affected by indoor environments and multipath phenomenon. Thus, the accuracy is very difficult to improve. In this paper, we put forward a method, which is able to leverage various other resources in localization. Besides the traditional RSS information, the environmental physical features, e.g., the light, temperature, and humidity information, are all utilized for localization. After building a comprehensive fingerprint map for the above information, we propose an algorithm to localize the target based on Naïve Bayesian. Experimental results show that the successful positioning accuracy can dramatically outperform traditional pure RSS-based indoor localization method by about $39 \%$. Our method has the potential to improve all the radio frequency (RF) based localization approaches.
\end{abstract}

\section{Introduction}

It is widely accepted that indoor localization is essential to many service applications and attracts many researchers' attentions [1-6]. For example, indoor navigation is helpful for the customer to find the path to the destination in a large shopping mall. In a hospital building, the patients can be easily found and taken care if indoor localization technologies are applied. In the underground parking place, indoor localization is useful for people to find their vehicles.

Among various indoor localization technologies [7-22], the technologies based on the Radio Signal Strength (RSS) are popular, since RSS can be easily obtained by common wireless devices (e.g., wireless sensors, mobile phone) without additional hardware. However, RSS are apt to be affected by indoor environments, since radio signal is easily reflected, refracted, and scattered by various indoor objects [23]. Therefore, signal emitted from one transmitter will arrive at the receiver from many different propagation paths. Such phenomenon is called multipath phenomenon. As a result, although many improvement works have been proposed, the localization accuracy based on such technologies is very difficult to improve.
In order to overcome this drawback, we put forward an approach, which is able to leverage various other resources in localization. In detail, besides the traditional RSS information, the environmental physical features, e.g., the light, temperature, and humidity, are all comprehensively utilized in localization. At the same time, no much additional cost is introduced.

The basic idea is to construct a comprehensive map [5, 24-26] named RTHL (means the RSS, temperature, humidity, and light), which not only covers the fingerprint of RSS information, but also contains the fingerprint of temperature, humidity, and light information from different places in the target area. Figure 1 shows an example of our basic idea. There are some reference sensor nodes (marked as $1,2, \ldots$, 6) hung on the ceiling acting as the transmitter. The tracking node on the ground acts as the receiver. At the beginning of training phase, we will construct the RTHL map, where the RSS, light intensity, temperature, and humidity information by the receiver at different places are all collected. In the online phase, we may utilize our localization algorithm based on Naïve Bayesian to accurately localize the target object.

Our experiments are based on 7 telosB [27] sensors, which are placed randomly in an $8 \times 10 \mathrm{~m}^{2}$ laboratory. 


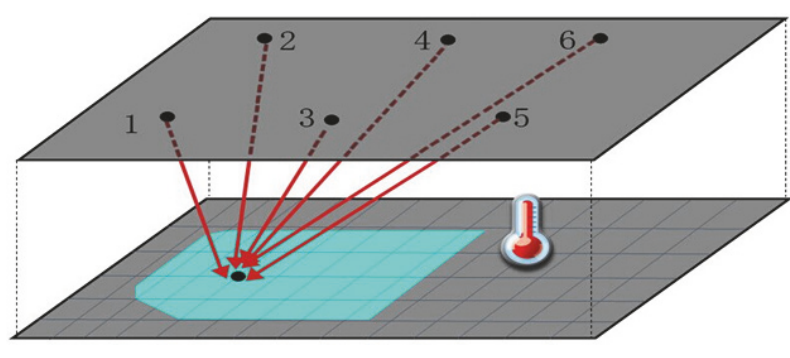

Figure 1: Basic idea.

Experimental results show us that the successful localization accuracy can dramatically outperform traditional indoor localization method based on just RSS by about $39 \%$.

The main contributions of this paper are as follows. First, we introduce different environmental physical features to improve the localization accuracy of traditional pure RSSbased indoor localization. Second, we propose one localization algorithm which can accurately localize the target. Third, we comprehensively perform the experiments in different indoor environments with low cost. At last but not least, our method is a general method, which has the potential to improve all the RF-based localization approaches.

The rest of this paper is organized as follows. In the next section, we will introduce the related work about localization technologies. In the following, we will describe our methodology in detail. Section 4 will present our localization system implementation and evaluate its performance. Finally, we will conclude this work and point out some possible future work directions.

\section{Related Work}

There are a large number of indoor localization technologies utilizing RSS [1-3, 19-21] or CSI information [22, 28-32]. RSS information can be easily obtained from common wireless devices, e.g., wireless sensors and smart mobile phone, since it is a kind of free source and can be obtained without any cost. Channel State Information (CSI) can also be collected if physical layer information can be visited.

For RSS-based technologies, Bluetooth $[16-18,33,34]$ is usually embedded in mobile phones, personal digital assistants (PDA), laptops, and other portable electronic devices, and the localization accuracy is limited. WLAN $[1,11,26]$ have been deployed in many public places, such as hospitals and universities. A large number of fingerprinting technologies are proposed accordingly to improve the localization accuracy. ZigBee technologies $[2,24,35]$ are able to localize the target through the mutual communication between sensors. Among these technologies, plenty of methods are adopted, including the machine learning algorithms and optimization algorithms, to improve the localization accuracy. However, since the radio signal is easily affected the by multipath phenomenon in indoor environments, the localization accuracy is difficult to be improved. Our work introduces other environmental features in localization, which is able to potentially improve all the above methods.
For CSI information [22, 31, 32], since it should visit the physical layer information, the device type is limited. WiFi devices are often selected in such technologies and special hardware interfaces are usually applied. However, in such technologies, CSI information is also easily affected by the multipath phenomenon in indoor environments. Our work can also improve the localization accuracy of such technologies.

There are also some other works introducing Inertial Measurement Unit (IMU) device. They utilize the accelerometer and Gyroscope information [36-40] from IMU in localization. However, such sensors are not available in all the devices. Furthermore, our work introduces more environment features, e.g., temperature, humidity, and light information, in localization. It can give an optional choice for some particular devices and improve the localization accuracy of those systems using IMU.

Luxapose [41] is explored for indoor positioning by using unmodified smartphones, it is required to slightly modify commercial LED luminaries. Moreover, it only utilizes one single environment feature (light intensity) in localization. If other environmental features are considered, the accuracy can be further enhanced. The SpyLoc [42] localization system leverages both acoustic and WiFi information in localization. However, if more environmental features such as ours are introduced, the localization accuracy can be further improved.

\section{Methodology}

In this section, we will first introduce the basic idea of our method. In the following, the detail algorithm to localize the target object will be described.

3.1. Basic Idea. In the subsection, we will show how the physical features can help in localization. We perform an initial experiment based on just one pair of transmitter and receiver (both TelosB sensors). The receiver is placed in different positions on the ground with different distance to the transmitter. Since the TelosB sensors are integrated with the light, temperature, and the humidity sensorsiij Nwe are able to collect the RSS plus the light intensity, temperature, and the humidity information from the receiver sensor.

As shown in Figure 2, we can observe that, at different positions on the ground, the RSS, light intensity, temperature, and the humidity information all have variance. Therefore, if we can leverage such variance of both RSS and physical features, we may improve the localization accuracy based on just RSS information.

3.2. Building the RTHL Map. Since we will utilize the RSS, temperature, humidity, and light intensity information (RTHL) together to localize the target, we should construct an RTHL map during the offline phase. At first, we hang a number of transmitters (e.g., 4, we will discuss how to set such value in the later subsection) on the ceiling. A receiver is placed at different places on the ground to collect its RTHL information. Such information will be transmitted back to the server. At each place on the ground, we can get a set of 


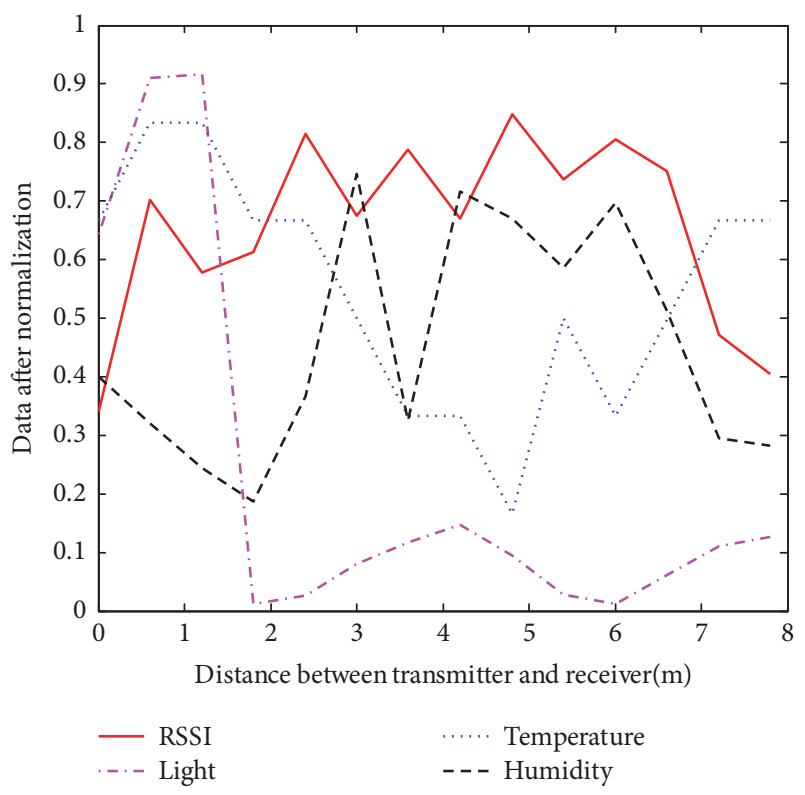

FIGURE 2: Observation based on one pair of transmitter and receiver.

vector $A_{i}=\left(A_{i 1}, A_{i 2}, \ldots, A_{i n}\right), i \in(1, m)$, where the value of $n$ depends on the number of reference nodes on the ceiling plus 3 physical feature values (the receiver's light, temperature, and humidity) and $m$ is the number of known places in this environment. Considering the variance of different RSS and physical feature information, for each attribute $A_{i j}, i \in(1, m)$, $j \in(1, n)$, we run a Min-Max normalization to eliminate the variance; then RTHL map can be built.

3.3. Naïve Bayesian Algorithm. After the RTHL map is built, in the online phase, when the target acting as the receiver appears in the same environment, the localization algorithm is able to be performed based on the RTHL map. The detail algorithm will be introduced below.

In our system, using the Naïve Bayesian classification, we see the different locations in RTHL map which have known coordinates as one category $C_{i}$, supposing there are $m$ locations, $i \in(1, m)$. When the tracking node enters the environment, it will receive the RSS information from different reference nodes, as well the sensed local temperature, humidity, and light intensity information. All this information is represented by a vector $X=\left(x_{1}, x_{2}, \ldots, x_{n}\right)$, where the value of $n$ is the number of reference nodes on the ceiling plus 3 physical feature values. The localization algorithm basically can be divided into the following 2 steps.

Step 1. Calculate $P\left(C_{1} / X\right), P\left(C_{2} / X\right), \ldots, P\left(C_{i} / X\right), P\left(C_{m} / X\right)$, $i \in(1, m)$, where $P\left(C_{i} / X\right)$ is the probability of $C_{i}$ given $X$.

Step 2. If $P\left(C_{k} / X\right)=\max \left\{P\left(C_{1} / X\right), P\left(C_{2} / X\right), \ldots, P\left(C_{k} / X\right)\right.$, ..., $\left.P\left(C_{m} / X\right)\right\}, k \in(1, m)$, we regard that the category of $X$ is $C_{k}$, which is the calculated target position.

The individual conditional probability in Step 1 can be calculated by the following.
(1) First, the categories of all items in the training sample set are already known. Therefore, we may get the conditional probability of each feature property in different categories by statistical methods, which are represented as

$$
\begin{gathered}
P\left(x_{1} / C_{1}\right), P\left(x_{2} / C_{1}\right), \ldots, P\left(x_{n} / C_{1}\right) \\
P\left(x_{1} / C_{2}\right), P\left(x_{2} / C_{2}\right), \ldots, P\left(x_{n} / C_{2}\right) \\
\ldots \\
P\left(x_{1} / C_{m}\right), P\left(x_{2} / C_{m}\right), \ldots, P\left(x_{n} / C_{m}\right) .
\end{gathered}
$$

(2) Since characteristic properties are independent of each in condition, we can obtain the following through the Naïve Bayesian theory:

$$
P\left(C_{i} / X\right)=\frac{P\left(X / C_{i}\right) P\left(C_{i}\right)}{P(X)}
$$

where $P\left(C_{i}\right)$ is the probability of category $C_{i}$ and $P(X)$ is the probability of $X$.

In formula (2), since the denominator is a constant for all categories, we just need the molecule to be maximized. Each property is independent on the condition; according to formula (2), we can know that

$$
\begin{aligned}
P & \left(X / C_{i}\right) P\left(C_{i}\right) \\
& =P\left(x_{1} / C_{i}\right) P\left(x_{2} / C_{i}\right) \cdots P\left(x_{n} / C_{i}\right) P\left(C_{i}\right) \\
& =P\left(C_{i}\right) \prod_{j=1}^{n} P\left(x_{j} / C_{i}\right)
\end{aligned}
$$

Therefore, with the known training samples and its categories, we can calculate the results of formula (3). According to the maximum value, we may determine which category $X$ belongs to. Since the signal intensity is continuously distributed in the indoor space, the value of each feature property obeys the Gauss distribution:

$$
g(x, \eta, \sigma)=\frac{1}{\sigma \sqrt{2 \pi}} e^{-(x-\eta)^{2} / 2 \sigma^{2}}
$$

Here, we know that

$$
\begin{aligned}
P\left(x_{j} / C_{i}\right) & =g\left(x_{j}, \eta_{j}, \sigma_{j}\right) \\
\eta_{j} & =\frac{1}{m} \sum_{i=1}^{m} x_{j i} \\
\sigma_{j} & =\sqrt{\frac{1}{m-1} \sum_{i=1}^{m}\left(x_{j i}-\eta_{j}\right)^{2}} \quad j \in(1, n)
\end{aligned}
$$

Therefore, using formulas (3), (4), and (5), we can get the category of $X$. Since we know the coordinates of each category in advance, the coordinates of the tracking node can be obtained. 


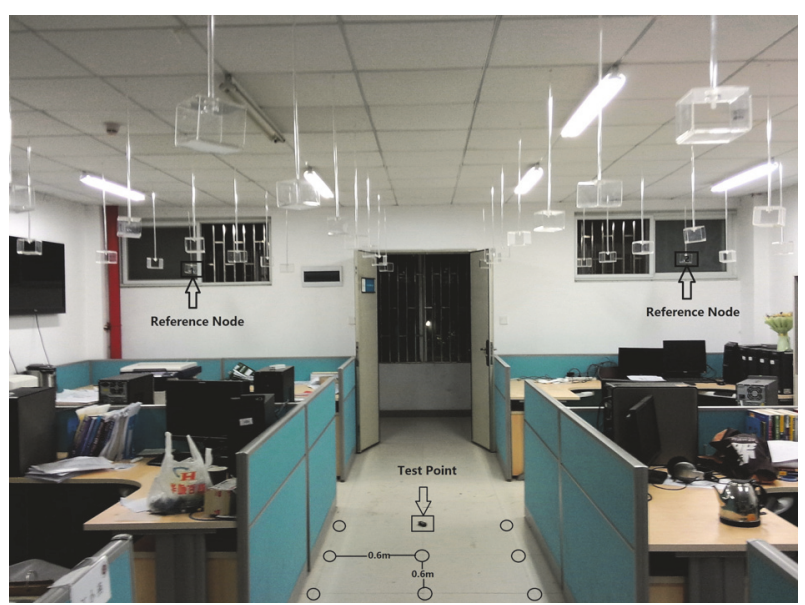

FIgURE 3: Experimental environment.

\section{Experiment and Evaluation}

In this section, we first introduce the experimental environment of our system and then show the performance evaluation.

4.1. Experiment Setup. We carry out the experiments in our laboratory, whose area is $8 \times 10$ square meters, as shown in Figure 3. We utilize telosB sensor as the wireless node, which is composed of CC2420 radio chips and MSP430 microcontroller [43]. Such sensor is able to get the RSS information from other sensors, light intensity, humidity, and temperature from its local area. We program 6 sensors as reference nodes to broadcast beacons periodically. The reference nodes are hung on the ceiling. The default transmission power is $0 \mathrm{dBm}$. The default channel is 11 . The tracking node on the ground acts as the receiver.

At first, in the offline phase, we build a RTHL fingerprint map consisting of RSS from the reference nodes, temperature, humidity, and the light intensity information of the whole laboratory environment. Later in the online phase, when the tracking node comes into the environment, it receives the RSS information from the reference nodes. It can sense its local temperature, humidity, and the light intensity information and transmit them back to the sink. The server runs our localization algorithm to calculate the position of the tracking node based on the RTHL map.

4.2. The Impact of the Number of Reference Nodes. At first, in order to investigate how the number of reference nodes will influence the localization results, we perform our experiments based on different number of reference nodes from 3 to 6 . We choose 3 as the initial test number, since 3 is the minimum number of reference nodes in most fingerprintbased localization algorithms.

As Figure 4 shows, we can see that, when the reference node number is chosen as $3,4,5$, and 6 , the average errors are $2.05 m, 2.11 \mathrm{~m}, 2.18 \mathrm{~m}$, and $1.96 \mathrm{~m}$, respectively. We find that, in general, when the number of reference nodes increases, the localization error will decrease. When the number of

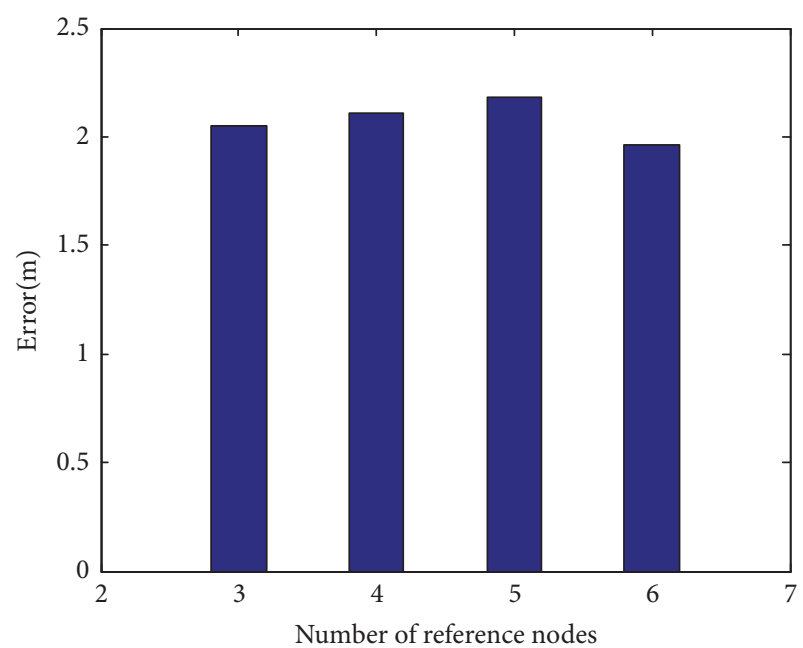

FIGURE 4: Comparison of different number of reference nodes.

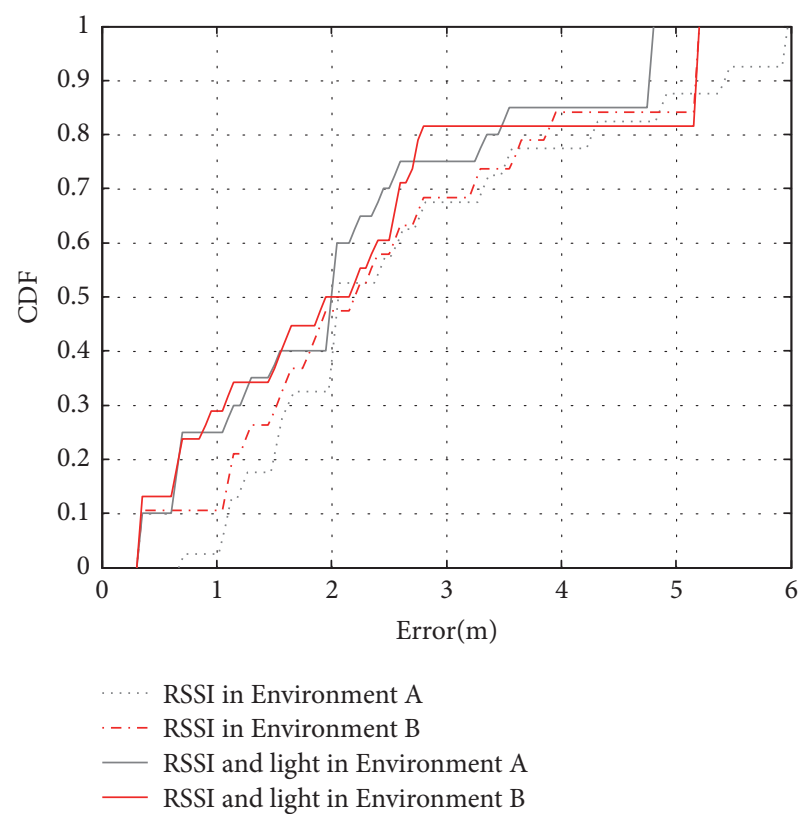

FIGURE 5: The impact of the light intensity.

reference nodes is set as 6 , the localization error is the smallest.

Therefore, in our later experiments we use 6 reference nodes in our setting.

4.3. The Impact of the Light Intensity. In this subsection, we will study how much the light intensity feature will improve the localization accuracy. Concerning it is a time-varying factor, and the light intensity information usually is different in the daytime and nighttime, we perform our experiments in such two scenarios. Environment A represents the daytime in our laboratory, while Environment B represents the nighttime in the same place. Based on 40 test samples, Figure 5 gives the experimental results. We find that the localization accuracy of leveraging light intensity information is much 
better than just using RSS information. In the daytime, the localization accuracy is improved by more than $15 \%$, which is possible due to the following reason. Since during the daytime the environment usually is filled with sunlight, the place in the lab close to the window usually has higher light intensity information. Therefore, the light intensity may vary a lot for different lab area. However, during the nighttime, the light from the lamp is usually uniformly distributed which results in the light intensity information having no big difference among different places. Usually larger variance in the RTHL fingerprint map will contribute more on the localization accuracy.

In summary, we find that, when leveraging light intensity information, the localization accuracy can be improved. If the environment has enough light intensity variance in different places, the localization accuracy can be further improved by about $30 \%$.

4.4. The Impact of the Temperature. In this subsection, we will investigate how much the indoor temperature will improve the localization accuracy. We also perform our experiment in both day and nighttime. Based on 40 test samples, the experimental result is shown in Figure 6. We find that, with the help of temperature information, the localization accuracy can be improved by about $10 \%$ for both day and night environments. Also we observe that, no matter in the day or night environment, the improvement of localization accuracy has no big difference for the two environments. The reason is possible that indoor area usually has air conditioner. Therefore, there is little temperature difference among difference places. Only the places close to the air conditioner may have lower temperature than the other places.

In conclusion, we know that the temperature information can improve the localization accuracy by about $10 \%$. If in other indoor area with higher temperature variance among different places, we believe the localization accuracy can be further improved.

4.5. The Impact of the Humidity. In this subsection, we will explore how much the indoor humidity will improve the localization accuracy. We also carry out our experiment in both day and nighttime. Based on 40 test samples, we can see the experimental result from Figure 7. It shows the humidity information can help to improve the localization accuracy by around 10\%, no matter in Environment A or Environment B. The reason that why the humidity does not improve the localization accuracy dramatically may be that common indoor area has air conditioner, which results in the humidity information having no big difference in different lab area.

To sum up, the humidity information is able to improve the localization accuracy. We believe that, if in an area where the humidity has a big difference at different places, the localization accuracy can be further improved.

4.6. Localization Accuracy. In this subsection, we utilize RSS and all the environmental factors (light intensity, temperature, and humidity) in localization. The accuracy of traditional method using just RSS is $1.96 m$, while our system

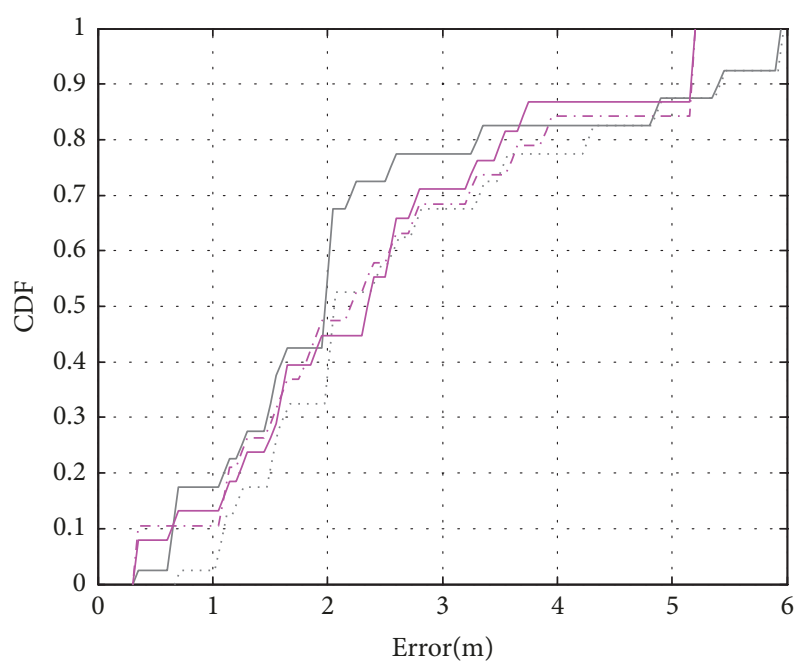

RSSI in Environment A

RSSI in Environment B

_ RSSI and temperature in Environment A

— RSSI and temperature in Environment B

FIgURE 6: The impact of temperature.

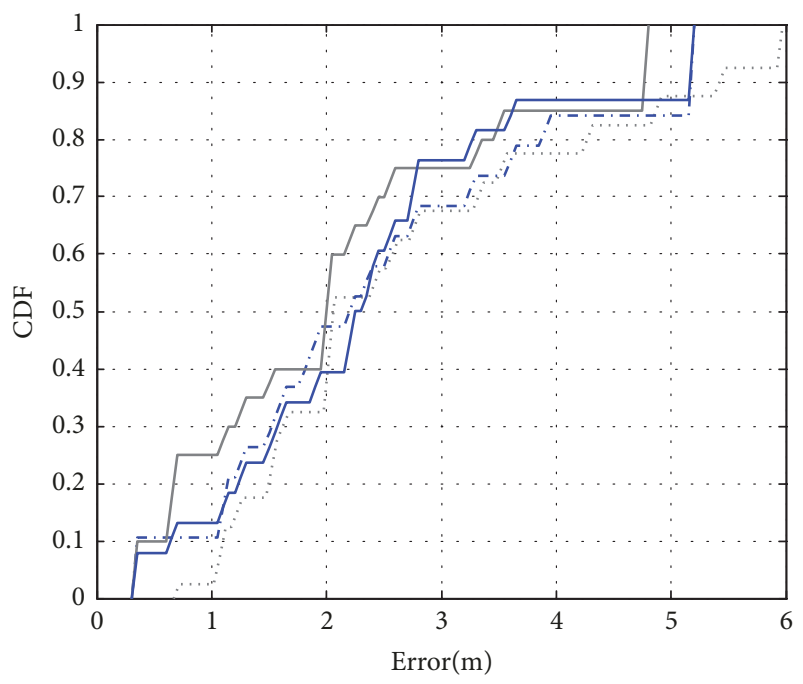

..... RSSI in Environment A

. . . - RSSI in Environment B

- RSSI and humidity in Environment A

— RSSI and humidity in Environment B

FIGURE 7: The impact of humidity.

is $1.19 \mathrm{~m}$, whose accuracy comparison is shown in Figure 8 . The algorithm running result is shown in Figure 9. The blue stars with number are the real positions of the tracking node, and the red stars with number are the calculated positions by using our system.

4.7. Latency. The latency of our system mainly depends on the beacon interval of each node. In our experiment, in order 


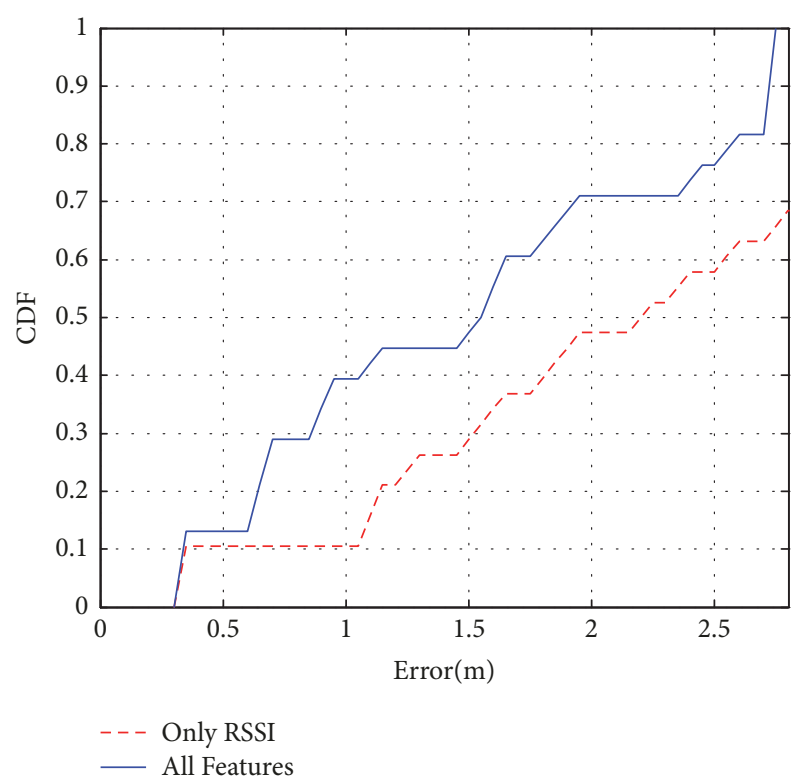

FIgURE 8: Accuracy comparison (CDF).

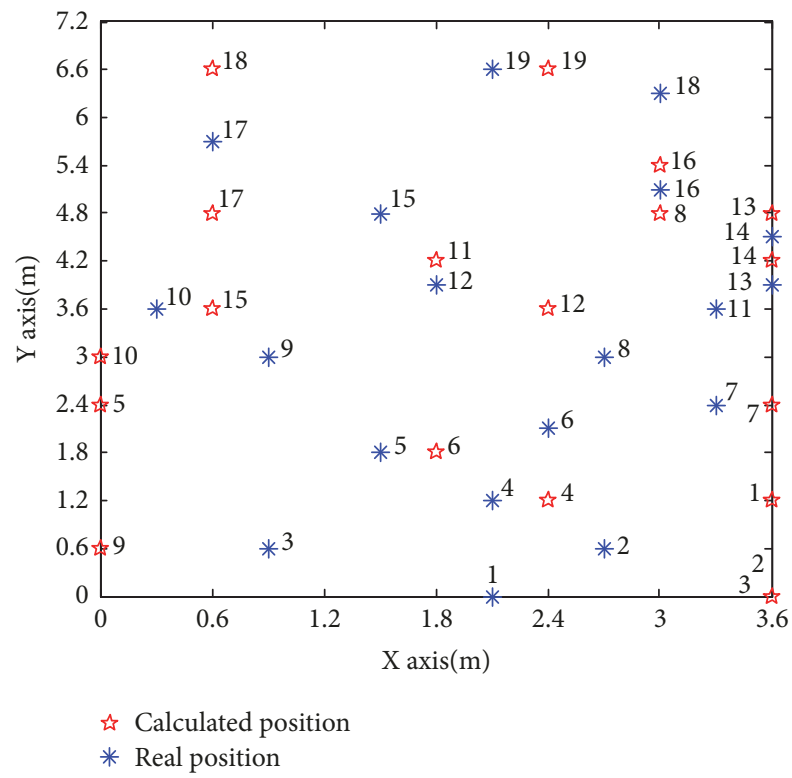

FIGURE 9: Localization result.

to avoid collision during data transmission, which causes data packets missing, we set the reference node's broadcast interval as $0.2 s$ to transmit a packet with 51 bytes. The other time, e.g., algorithm running time in the server part, is neglected. Therefore, the latency is about $0.2 s$.

4.8. Discussion. Actually in our approach, how much the localization accuracy will be improved depends on different environments. Our experiments are performed in a relatively closed area. Regarding different factors, we have the following suggestions.
In such areas, the humidity of the whole area is relatively uniform, which results in the humidity feature having less impact on localization accuracy. We believe that if in a different large indoor complex environment, the improvement could be larger.

For the temperature feature, in our environment, the temperature close to the air conditioning is relatively low. We believe these features will contribute a lot to those closed environments where the air conditioning is always on.

Regarding light intensity feature during night in our environment, where artificial lights are used, the light intensity is different at different places. For example, a place close to a chair or under desk has a weak light intensity. Such feature will contribute a lot in an environment if the environment does not have relatively constant source of light. However, if in an open area which has changing light source, e.g., the sun light in the daytime, we suggest not using such feature in localization. It is also the reason why light intensity does not contribute too much during the daytime to our experiment, since our lab has many open windows.

To sum up, our approach gives a general solution to introducing more environment features in localization. How to choose these features in a specific environment depends on the detail condition in such environment. Users may choose those features which will contribute the most in localization. We believe our approach is able to potentially improve all the RSS-based localization technologies.

\section{Conclusion and Future Work}

In this paper, we have proposed a method, which can improve traditional RSS-based indoor localization accuracy by leveraging various environmental physical features, e.g., the light, temperature, and humidity information. By building a comprehensive fingerprint map for the above information, Naïve Bayesian algorithm is used to localize the target. We implement our system in two different environments based on wireless sensor networks. Experimental results show that, compared with the traditional indoor localization approach based on just RSS information, our method based on Naïve Bayesian can improve the localization accuracy by about $39 \%$.

As future work, at first, we will try our approach in a more complicated large indoor area. Thus, the environmental features may vary a lot at different places, which may increase the localization accuracy. Furthermore, we may use higher precision light, temperature, and humidity sensors to do experiments to achieve higher accuracy. At last, we will try our system in a $3 D$ area.

\section{Data Availability}

The data used to support the findings of this study are available from the corresponding author upon request.

\section{Conflicts of Interest}

The authors declare that they have no conflicts of interest. 


\section{Acknowledgments}

This research was supported in part by Shenzhen Peacock Talent Grant 827-000175 and China NSFC Grants 61202377 and U1301251. Dian Zhang is the corresponding author.

\section{References}

[1] M. Kotaru, K. Joshi, D. Bharadia et al., "SpotFi: Decimeter Level Localization Using WiFi," Acm Sigcomm Computer Communication Review, vol. 45, no. 5, pp. 269-282, 2015.

[2] D. Zhang, J. Ma, Q. Chen, and L. M. Ni, "An RF-based system for tracking transceiver-free objects," in Proceedings of the 5th Annual IEEE International Conference on Pervasive Computing and Communications (PerCom '07), pp. 135-144, March 2006.

[3] X. Guo, D. Zhang, K. Wu, and L. M. Ni, "MODLoc: Localizing multiple objects in dynamic indoor environment," IEEE Transactions on Parallel and Distributed Systems, vol. 25, no. 11, pp. 2969-2980, 2014.

[4] C. Wu, Z. Yang, Y. Liu et al., "WILL: Wireless Indoor Localization without Site Survey," IEEE Transactions on Parallel and Distributed Systems, vol. 24, no. 4, pp. 839-848, 2013.

[5] S. Sorour, Y. Lostanlen, S. Valaee et al., "Joint Indoor Localization and Radio Map Construction with Limited Deployment Load," Mobile Computing IEEE Transactions, vol. 14, no. 5, pp. 1031-1043, 2013.

[6] S.-H. Jung, B.-C. Moon, and D. Han, "Unsupervised learning for crowdsourced indoor localization in wireless networks," IEEE Transactions on Mobile Computing, vol. 15, no. 11, pp. 28922906, 2016.

[7] R. Mautz, "Indoor Positioning Technologies," Südwestdeutscher Verlag für Hochschulschriften, 2012.

[8] D. Yang, H. Gonzalez-Banos, and L. Guibas, "Counting people in crowds with a real-time network of simple image sensors," IEEE International Conference on Computer Vision, vol. 1, pp. 122-129, 2003.

[9] Q. Cai and J. Aggarwal, "Automatic tracking of human motion in indoor scenes across multiple synchronized video streams," in Proceedings of the International Conference on Computer Vision, pp. 356-362, 2002.

[10] T. Kivimäki, T. Vuorela, P. Peltola, and J. Vanhala, "A review on device-free passive indoor positioning methods," International Journal of Smart Home, vol. 8, no. 1, pp. 71-94, 2014.

[11] M. Huber, F. Kamangar, and I. Chlamtac, "Indoor location tracking using RSSI readings from a single Wi-Fi access point," Wireless Networks, vol. 13, no. 2, pp. 221-235, 2007.

[12] Y. Wang, J. Liu, Y. Chen, M. Gruteser, J. Yang, and H. Liu, "E-eyes: Device-free location-oriented activity identification using fine-grained WiFi signatures," in Proceedings of the 20th ACM Annual International Conference on Mobile Computing and Networking, MobiCom 2014, pp. 617-628, USA, September 2014.

[13] J. S. Lee, Y. W. Su, and C. C. Shen, "A comparative study of wireless protocols: bluetooth, UWB, ZigBee, and Wi-Fi," in Proceedings of the 33rd Annual Conference of the IEEE Industrial Electronics Society (IECON '07), pp. 46-51, 2008.

[14] C. S. Jensen, H. Lu, and B. Yang, "Graph model based indoor tracking," in Proceedings of the 10th International Conference on Mobile Data Management: Systems, Services and Middleware (MDM '09), pp. 122-131, May 2009.
[15] C. Jensen S, H. Lu, and B. Yang, "Indexing the trajectories of moving objects in symbolic indoor space," in Advances in Spatial and Temporal Databases, vol. 5644 of Lecture Notes in Computer Science, pp. 208-227, Springer, Berlin, Germany, 2009.

[16] S. Feldmann, K. Kyamakya, A. Zapater et al., "An Indoor Bluetooth-Based Positioning System: Concept, Implementation and Experimental Evaluation," in Proceedings of the International Conference on Wireless Networks, Icwn '03, pp. 109-113, 2003.

[17] Y. Gu, L. Quan, F. Ren, and J. Li, "Fast indoor localization of smart hand-held devices using bluetooth," in Proceedings of the 10th IEEE International Conference on Mobile Ad-Hoc and Sensor Networks, MSN '14, pp. 186-194, 2015.

[18] J. S. Lee, Y. W. Su, and C. C. Shen, "A comparative study of wireless protocols: bluetooth, UWB, ZigBee, and Wi-Fi," in Proceedings of the 33rd Annual Conference of the IEEE Industrial Electronics Society (IECON '07), pp. 46-51, Taipei, Taiwan, November 2007.

[19] X. Ye, Y. Wang, W. Hu, L. Song, Z. Gu, and D. Li, "WarpMap: Accurate and Efficient Indoor Location by Dynamic Warping in Sequence-Type Radio-Map," in Proceedings of the 2016 13th Annual IEEE International Conference on Sensing, Communication, and Networking (SECON), pp. 1-9, London, United Kingdom, June 2016.

[20] M. N. Husen and S. Lee, "High Performance Indoor Location Wi-Fi Fingerprinting using Invariant Received Signal Strength," Husen2016High, pp. 1-6, 2016.

[21] Y. Shu, Y. Huang, J. Zhang et al., "Gradient-based fingerprinting for indoor localization and tracking," IEEE Transactions on Industrial Electronics, vol. 63, no. 4, pp. 2424-2433, 2016.

[22] X. Wang, L. Gao, S. Mao, and S. Pandey, "CSI-Based Fingerprinting for Indoor Localization: A Deep Learning Approach," IEEE Transactions on Vehicular Technology, vol. 66, no. 1, pp. 763-776, 2017.

[23] “various indoor objects," http://en.wikipedia.org/wiki/Indoor_ positioning_system.

[24] P. Bahl and V. N. Padmanabhan, "RADAR: an in-building RFbased user location and tracking system," in Proceedings of the 19th IEEE Annual Joint Conference of the IEEE Computer and Communications Societies (INFOCOM '00), vol. 2, pp. 775-784, IEEE, Tel Aviv, Israel, March 2000.

[25] Y. Shu, P. Coue, Y. Huang, J. Zhang, P. Cheng, and J. Chen, "GLoc: Indoor localization leveraging gradient-based fingerprint map," in Proceedings of the IEEE INFOCOM 2014 - IEEE Conference on Computer Communications Workshops (INFOCOM WKSHPS), pp. 129-130, Toronto, ON, Canada, April 2014.

[26] M. Youssef and A. Agrawala, "The Horus WLAN location determination system," in Proceedings of the 3rd International Conference on Mobile Systems, Applications, and Services (MobiSys '05), pp. 205-218, ACM, June 2005.

[27] http://www.memsic.com/userfiles/files/Datasheets/WSN/telosb_ datasheet.pdf.telosB.

[28] K. Wu, J. Xiao, Y. Yi et al., "CSI-Based Indoor Localization," IEEE Transactions on Parallel Distributed Systems, vol. 24, no. 7, pp. 1300-1309, 2013.

[29] K. Wu, J. Xiao, Y. Yi et al., "FILA: Fine-grained indoor localization," in Proceedings of the IEEE Conference on Computer Communications (INFOCOM '12), vol. 131, pp. 2210-2218, March 2012.

[30] J. Xiao, K. Wu, Y. Yi et al., "Pilot: passive device-free indoor localization using channel state information," in Proceedings of 
the IEEE 33rd International Conference on Distributed Computing Systems (ICDCS '13), pp. 236-245, July 2013.

[31] K. Ali, A. X. Liu, W. Wang, and M. Shahzad, "Keystroke recognition using WiFi signals," in Proceedings of the 21st Annual International Conference on Mobile Computing and Networking, MobiCom 2015, pp. 90-102, France, September 2015.

[32] W. Wang, A. X. Liu, M. Shahzad et al., "Understanding and modeling of WiFi signal based human activity recognition," in Proceedings of the 21st Annual International Conference on Mobile Computing and Networking, MobiCom 2015, pp. 65-76, September 2015.

[33] M. Altini, D. Brunelli, E. Farella, and L. Benini, "Bluetooth indoor localization with multiple neural networks," in Proceedings of the 5th International Symposium on Wireless Pervasive Computing (ISWPC '10), pp. 295-300, IEEE, Modena, Italy, May 2010.

[34] Y. Wang, Q. Ye, J. Cheng, and L. Wang, "RSSI-Based Bluetooth Indoor Localization," in Proceedings of the 11th International Conference on Mobile Ad-Hoc and Sensor Networks, MSN 2015, pp. 165-171, December 2015.

[35] J. Larranaga, L. Muguira, J. Lopez-Garde et al., "An environment adaptive ZigBee-based indoor positioning algorithm," in Proceedings of the International Conference on Indoor Positioning and Indoor Navigation, pp. 1-8, 2010.

[36] H. Lategahn, M. Schreiber, J. Ziegler, and C. Stiller, "Urban localization with camera and inertial measurement unit," in Proceedings of the 2013 IEEE Intelligent Vehicles Symposium, IEEE IV 2013, pp. 719-724, aus, June 2013.

[37] Y. Nam, "Map-based indoor people localization using an inertial measurement unit," Journal of Information Science and Engineering, vol. 27, no. 4, pp. 1233-1248, 2011.

[38] C.-H. Hsu and C.-H. Yu, "An Accelerometer based approach for indoor localization," in Proceedings of the Symposia and Workshops on Ubiquitous, Autonomic and Trusted Computing in Conjunction with the UIC'09 and ATC'09 Conferences, UIC-ATC 2009, pp. 223-227, aus, July 2009.

[39] J. Bird and D. Arden, "Indoor navigation with foot-mounted strapdown inertial navigation and magnetic sensors," IEEE Wireless Communications Magazine, vol. 18, no. 2, pp. 28-35, 2011.

[40] H. Myung, H. Lee, K. Choi et al., "Mobile robot localization with gyroscope and constrained Kalman filter," in Proceedings of the IEEE/RSJ International Conference on Intelligent Robots and Systems, pp. 442-447, 2010.

[41] Y.-S. Kuo, P. Pannuto, K. Hsiao et al., Luxapose: Indoor Positioning with Mobile Phones And Visible Light, 2014.

[42] M. Uddin and T. Nadeem, "SpyLoc: a light weight localization system for smartphones," Fuzzy Sets and Systems, vol. 103, no. 2, pp. 303-315, 2013.

[43] A. Milenković, C. Otto, and E. Jovanov, "Wireless sensor networks for personal health monitoring: issues and an implementation," Computer Communications, vol. 29, no. 13-14, pp. 2521-2533, 2006. 


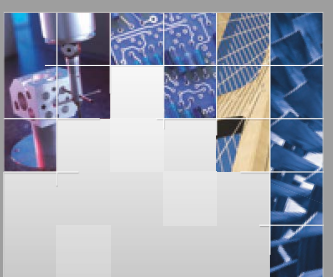

\section{Enfincering}
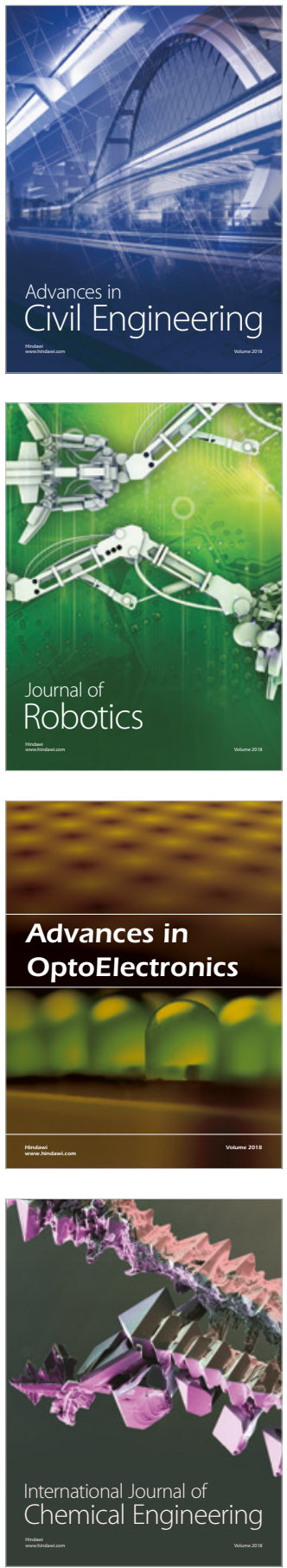

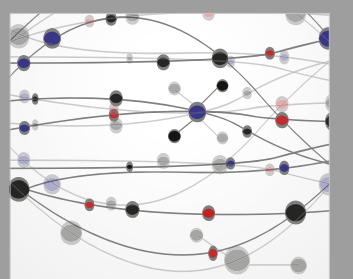

\section{Rotating \\ Machinery}

The Scientific World Journal

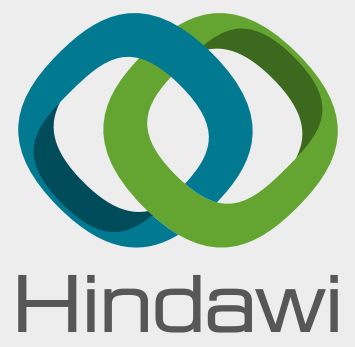

Submit your manuscripts at

www.hindawi.com
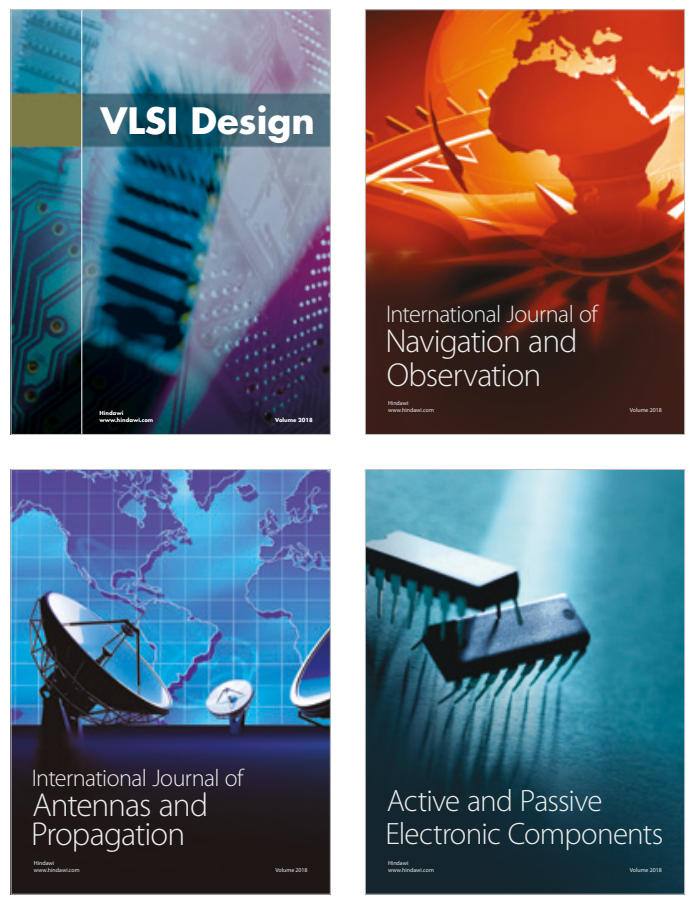
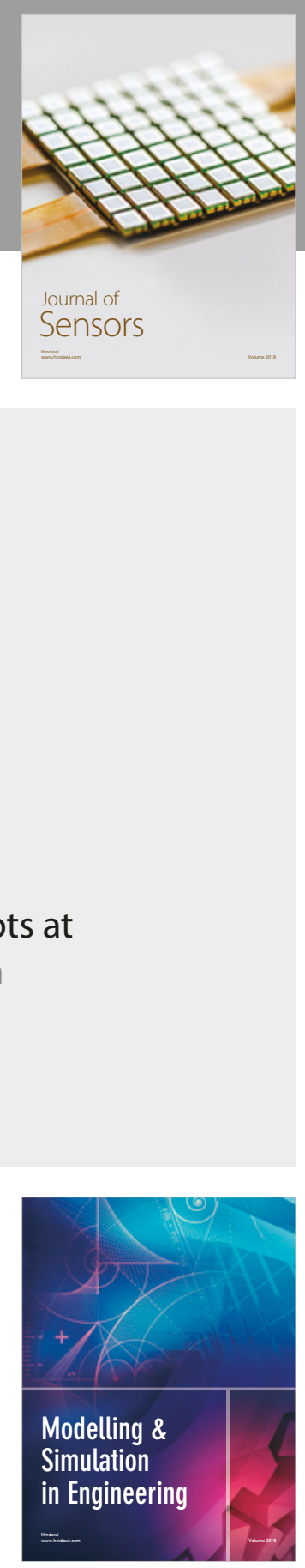

\section{Advances \\ Multimedia}
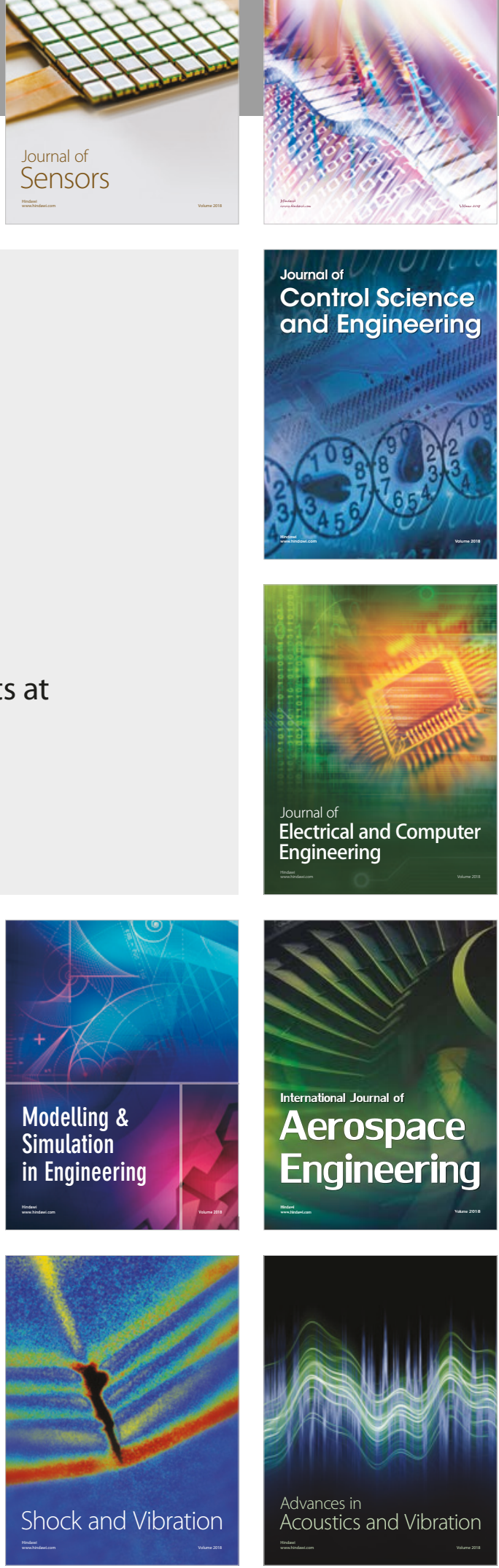